\title{
Non-Subsampled Shearlet Transform and Log-Transform Methods for Despeckling of Medical Ultrasound Images
}

\author{
Reza ABAZARI ${ }^{1 *}$, Mehrdad LAKESTANI ${ }^{2}$ \\ ${ }^{1}$ Department of Mathematics, Faculty of Sciences, University of Mohaghegh Ardabili, \\ Ardabil, Iran \\ ${ }^{2}$ Department of Applied Mathematics, Faculty of Mathematical Sciences, \\ University of Tabriz, Tabriz, Iran \\ e-mail:abazari-r@uma.ac.ir
}

Received: September 2017; accepted: November 2018

\begin{abstract}
Medical Ultrasound is a diagnostic imaging technique based on the application of ultrasound in various branches of medical sciences. It can facilitate the observation of structures of internal body, such as tendons, muscles, vessels and internal organs such as male and female reproductive system. However, these images usually degrade by a special kind of multiplicative noise called speckle. The main effects of speckle noise in the ultrasound images appear in the edges and fine details which lead to reduce their resolution and consequently make difficulties in medical diagnosing. Therefore, reducing of speckle noise seriously plays an important role in image diagnosing. Among the various methods that have been proposed to reduce the speckle noise, there exists a class of approaches that firstly convert multiplicative speckle noise into additive noise via log-transform and secondly perform the despeckling process via a directional filter. Usually, the additive noises are mutually uncorrelated and obey a Gaussian distribution. On the other hand, non-subsampled shearlet transform (NSST), as a multi scale method, is one of the effective methods in image processing, specially, denoising. Since NSST is shift invariant, it diminishes the effect of pseudo-Gibbs phenomena in the denoising. In this paper, we describe a simple image despeckling algorithm which combines the log-transform as a pre-processing step with the non-subsampled shearlet transform for strong numerical and visual performance on a broad class of images. To illustrate the efficiency of the proposed approach, it is applied on a sample image and two real ultrasound images. Numerical results illustrate that the proposed approach can obtain better performance in term of peak signal to noise ratio (PSNR) and structural similarity (SSIM) index rather than existing state-of-the-art methods.
\end{abstract}

Key words: ultrasound image, discrete shearlet transform, non-subsampling, log-transform, speckle noise.

\section{Introduction}

The invention of the computational tools, especially computers, in the recent decades has led to produce many new medical imaging techniques, such as computed tomography scan

\footnotetext{
* Corresponding author.
} 
(CT scan), ultrasound imaging, magnetic resonance imaging (MRI) and etc., for medical diagnosing. Among these imaging techniques, the ultrasound imaging technique is popularly used in medical diagnosing, mainly in obstetrics, gastrointestinal and cardiovascular fields. The main reasons for the popularity of ultrasound imaging technique are related to other more sophisticated imaging techniques, such as CT, MRI or Positron Emission Tomography (PET), and are its low cost, non-ionizing properties and its portability to provide real time interactive visualization of anatomical structures (Abbott and Thurstone, 1979). Ultrasound images are created by ultrasonic waves, which are produced by dispatching special sound waves through body tissues and receiving their reflex by a transducer and they are processed and transformed into a digital image (Ragesh et al., 2011). Usually, the inappropriate contact or being air gap between the transducer and body lead to create an interference pattern called speckle (Abbott and Thurstone, 1979). Speckle is a particular type of noise that is characterized by a granular pattern of bright and dark spots which tend to degrade the fine details and edges of ultrasound images and consequently lead to complication in clinical diagnosing.

Generally, two families of approaches have been proposed for reducing of speckle noise of ultrasound images:

A) The first family are those filters that perform on ultrasound images directly. In Abazari and Lakestani (2018a) the authors applied the Fourier based discrete shearlet transform to despeckle medical ultrasound images. Ritenour et al. (1984) applied the median filter to suppress speckle noise from the digital radiographic images. The adaptive weighted form of median filter is also suggested by Loupas et al. (1989) to denoise ultrasonic images. The wavelet transform and its complex form are also applied directly for ultrasound images in Deka and Bora (2013), Khare et al. (2010). Elyasi and Pourmina (2016) have employed the TV regularization with modified bayes shrink for reducing of speckle noise from ultrasound images. In Zong et al. (1998) it is shown that the linear filtering cannot be an optimal method for reducing the speckle noises. In Elmoniem et al. (2002) a denoising method based on nonlinear coherent diffusion (NCD) is utilized. Yu and Acton (2002) proposed a despeckle method based on anisotropic diffusion method (SRAD) to cast the spatial adaptive filers into diffusion model (Yu and Acton, 2002). Oriented version of SRAD (OSRAD) filter (Krissian et al., 2007), which is one of the extension of SRAD, was proposed with appraising the properties of the numerical scheme associated with SRAD filter. In Vese and Osher (2003) modified the TV minimization algorithm to reduce speckle noise from ultrasound images. Zhang et al. (2001) proposed an algorithm based on wavelet frame for denoising of Doppler ultrasound signals. Wang et al. (2014) also introduced a new denoising approach based on framelet regularization. An automated approach for segmentation of intravascular ultrasound images is also studied in Vard et al. (2012).

B) The second family are those that firstly convert the speckle noise to an additive noise via log-transform method and then a special filtering is applied to denoise the additive noise. The fundamental properties of speckle noises (Goodman, 1976), the logtransform speckle noise is studied in Hiremath et al. (2013) and their properties in the Contourlet Transform Domain are clearly explained in Kabir and Bhuiyan (2015). The discrete wavelet denoising approaches via log-transform method (Rajeshwar, 2018) are 
utilized for reducing the speckle noises of medical ultrasound images. In Hazarika et al. (2015), the enhanced Lee filter in lapped orthogonal transform (LOT) domain is applied to despeckle the log-transformed SAR images. Some other approaches to reduce speckle noise in medical ultrasound images are also proposed in Huang et al. (2016), Gupta et al. (2004), Bhuiyan et al. (2009) and the references given there.

As mentioned above, several methods have been proposed for reducing speckle noises, however, each method has its assumptions, advantages, and disadvantages. Among them, the methods based on wavelet transform have good efficiency in noise reduction. However, wavelets fail to capture the geometric regularity along the singularities of curves, because of their isotropic support. To exploit the anisotropic regularity of a curve along edges, the basis must include elongated functions that are nearly parallel to the edges. Several image representations have been proposed to capture the geometric regularity of a given image. Some of these representations are ridgelet (Candes, 1998), brushlet (Meyer and Ronald, 1997), curvelet (Candes and Donoho, 2000), beamlet (Donoho and Huo, 2001), contourlet (Do and Vetterli, 2005) and recently proposed shearlet (Labate et al., 2005).

The sheartlet transform as an alternative anisotropic multi-resolution system has been introduced by Labate $e t$ al. (2005) which yields nearly optimal approximation properties (Guo and Labate, 2007). Furthermore, the definition of shearlet transform is such that includes various scales, location and orientation in order to optimally represent an image. This new representation is based on a simple and rigorous mathematical framework which not only provides a more flexible theoretical tool for the geometric representation of multidimensional data, but is more natural for implementation. As a result, the shearlet approach can be associated to a multiresolution analysis (MRA) and this leads to a unified treatment of both the continuous and discrete world (Labate et al., 2005). Also, unlike the wavelets, they are optimal in sparse representation of multi-dimensional data (Guo and Labate, 2012) and, unlike curvelets, their directionality is achieved by shear matrices instead of rotation matrices (Guo and Labate, 2013). The shearlet transform has been applied in diverse areas of engineering and medical sciences, including inverse problems (Colonna et al., 2010), image separation (Kutyniok and Lim, 2011), image restoration (Patel et al., 2009), image denoising (Lakestani et al., 2016) and medical image analysis (Abazari and Lakestani, 2018a). However, due to shift variant nature of the shearlet, this method produces artifacts in the most of their process more in image denoising and image fusion (Vishwakarma et al., 2018). Some improved methods proposed to rectify the mentioned artifacts. Recently, the author proposed a hybrid denoising method based on shearlet transform and yaroslavsky's method (Abazari and Lakestani, 2018b) to suppress the effect of the pseudo-Gibbs phenomena and shearlet-like artifacts in denoising. The non-subsampled shearlet transform (NSST) is also proposed to capture edges and line discontinuities for image fusion (Vishwakarma et al., 2018). Since the NSST is shift invariant, it diminishes the effect of pseudo-Gibbs phenomena and shearlet-like artifacts in the related processing.

In this paper, by focusing on the non-subsampled form of "discrete shearlet transform" (Hou et al., 2012), we have proposed a despeckling approach via log-transform for speckle noisy ultrasound images. To illustrate the efficiency of the proposed approach, it 
is applied on a sample image and two real ultrasound images. Numerical results illustrate that the proposed approach can obtain better performance in terms of peak signal to noise ratio (PSNR) and structural similarity (SSIM) index rather than existing state-of-the-art methods.

\section{Speckle Noise}

Speckle noise is characterized by a peculiar granular pattern of bright and dark spots which lead to degrade the resolution of ultrasound images. This typical pattern is also observed in other kind of images involving coherent radiation, such as Laser and Synthetic Aperture Radar (SAR).

Generally, the speckle noise is described as a multiplicative phenomenon. Suppose that the observed image $f_{n}$ be degraded of noise free image $f$ by the speckle noise $n_{s}$ and an additive noise (such as thermal noise) $n_{a}$ as follows from Kabir and Bhuiyan (2015):

$$
f_{n}=f n_{s}+n_{a}
$$

Since the effect of additive noise in comparison to speckle noise is very little, so equation (1) can be written as

$$
f_{n}=f n_{s}
$$

By applying the log-transformation on (2), we obtain

$$
F_{n}=F+N_{s},
$$

where $F_{n}=\log \left(f_{n}\right), F=\log (f)$ and $N_{s}=\log \left(n_{s}\right)$. The probability density function (pdf) of $n_{s}$ is given by a Rayleigh pdf as follow:

$$
p d f\left(n_{s}\right)=\frac{n_{s}}{\alpha^{2}} e^{-\frac{n_{s}^{2}}{2 \alpha^{2}}},
$$

where $\alpha$ is the shape parameter and the expected value of $n_{s}$ will be $E\left(n_{s}\right)=\alpha \sqrt{\frac{\pi}{2}}$. From (3) and (4), it follows that

$$
p d f\left(N_{s}\right)=\frac{e^{N_{s}}}{\alpha^{2}} e^{-\frac{e^{2 N_{s}}}{2 \alpha^{2}}},
$$

where $p d f\left(N_{s}\right)$ is the pdf of $N_{s}$. By applying multi-resolution transform, such as shearlet transform, on (3), we obtain

$$
y=x+\varepsilon,
$$

where $y, x$ and $\varepsilon$ represents the coefficients corresponding to $F_{n}, F$ and $N_{s}$, respectively. In Goodman (1976), Goodman studied some fundamental properties of speckle noise. 
Also, he shows that the statistics of log-transformed speckle noise is given by a doubleexponential probability density function which is known as Fisher-Tippett probability density function. For more information about properties of speckle noise, please see Goodman (1976), Kabir and Bhuiyan (2015) and the references mentioned there.

\section{Shearlet Transform}

In this section, the shearlet and its transform both in continuous and discrete form will be briefly explained. The shearlet representation is a directional representation system that provides more geometrical information and shearlets are frame elements used in this representation scheme.

Definition 1. For any $\psi \in L^{2}\left(R^{2}\right)$, the continuous shearlet system is defined as follows

$$
\mathcal{S H}(\psi)=\left\{\psi_{a, s, t}(x)=a^{-\frac{3}{4}} \psi\left(A_{a}^{-1} S_{s}^{-1}(x-t)\right), a>0, s \in R, t \in R^{2}\right\},
$$

where $A_{a}=\left(\begin{array}{cc}a & 0 \\ 0 & \sqrt{a}\end{array}\right)$ is anisotropic dilation matrix as a mean to change the resolution and $S_{s}=\left(\begin{array}{ll}1 & s \\ 0 & 1\end{array}\right)$ is shear transformation matrix as a means to change the orientation.

The dilation matrix $A_{a}$ resembles the parabolic scaling, which has an elongated history in the literature of harmonic analysis and can be outlined back to the second dyadic decomposition from the theory of oscillatory integrals. Briefly, from the dilation matrix $A_{a}$ it can be concluded that the scaling in the $x$-direction is square of the scaling in the $y$-direction. The general form of dilation matrix $A_{a}$ is $A_{a}=\operatorname{diag}\left(a, a^{\alpha}\right)$ with the parameter $\alpha \in(0,1)$ that controls the degree of anisotropy, however, the value $\alpha=\frac{1}{2}$ plays a special role in the discrete setting. In fact, parabolic scaling is required in order to obtain optimally sparse approximations of cartoon-like images (see Definition 2), since it is best adapted to the $C^{2}$-regularity of the curves of discontinuity in the cartoon-like images class (Guo and Labate, 2007). The shearing matrix $S_{s}$ also parameterizes the orientations using the variables associated with the slopes rather than the angles, and has the advantage of leaving the integer lattice invariant, provided $s$ is an integer. The geometric effects of parabolic scaling and shearing with fixed parameter $a$ and several parameter $s$ are illustrated in Fig. 1. The associated continuous shearlet transform of any $f \in L^{2}\left(R^{2}\right)$ is given by

$$
\mathcal{S} \mathcal{H}_{\psi} f(a, s, t)=\left\langle f, \psi_{a, s, t}\right\rangle .
$$

In other words, $\mathcal{S} \mathcal{H}_{\psi}$ maps the function $f$ to the coefficients $\mathcal{S H}_{\psi} f(a, s, t)$ associated with the scale variable $a>0$, the orientation variable $s \in R$, and the location variable $t \in R^{2}$.

Now, our main aim is to achieve a continuous shearlet transform, which becomes an isometry, since this is automatically associated with a reconstruction formula. To do it, 


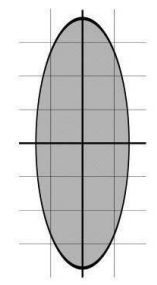

(a) $s=0$

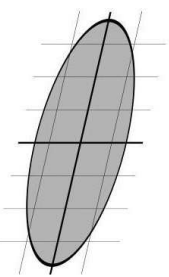

(b) $s=\frac{1}{4}$

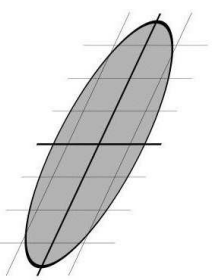

(c) $s=\frac{1}{2}$

Fig. 1. The geometric effects of parabolic scaling and shearing with fixed parameter $a$ and several parameter $s$. (a) $s=0$, (b) $s=\frac{1}{4}$ and (c) $s=\frac{1}{2}$.

the generating function $\psi$ must be a well localized function and be compatible with admissibility condition as follows from Labate et al., 2005,

$$
\int_{R^{2}} \frac{\left|\hat{\psi}\left(\xi_{1}, \xi_{2}\right)\right|^{2}}{\xi_{1}^{2}} d \xi_{2} d \xi_{1} \leqslant \infty
$$

So that, each $f \in L^{2}\left(R^{2}\right)$ has the representation

$$
f=\int_{R^{n}} \int_{-\infty}^{\infty} \int_{0}^{\infty}\left\langle f, \psi_{a, s, t}\right\rangle \psi_{a, s, t} \frac{d a}{a^{3}} d s d t .
$$

Consequently, it can be easily construct examples of shearlets, including examples of admissible shearlets which are well localized. Essentially any function $\psi$ such that $\hat{\psi}$ is compactly supported away from the origin is an admissible shearlet. A particular example of these representation is classical shearlet, wherein for any $\xi=\left(\xi_{1}, \xi_{2}\right) \in \widehat{R}^{2}, \xi_{1} \neq 0$, the generating function $\psi$ is setting such that

$$
\hat{\psi}\left(\xi_{1}, \xi_{2}\right)=\hat{\psi}_{1}\left(\xi_{1}\right) \hat{\psi}_{2}\left(\frac{\xi_{2}}{\xi_{1}}\right)
$$

where $\psi_{1} \in L^{2}(R)$ is a wavelet which satisfies in the Calderon condition (Guo and Labate, 2007), given by

$$
\sum_{j \in Z}\left|\hat{\psi}_{1}\left(2^{-j} \xi\right)\right|^{2}=1, \quad \forall \text { a.e. } \xi \in R
$$

with supp $\hat{\psi}_{1} \subset\left[-2,-\frac{1}{2}\right] \cup\left[\frac{1}{2}, 2\right]$ and $\psi_{2} \in L^{2}(R)$ is a bump function with supp $\hat{\psi}_{2} \subset$ $[-1,1]$ and

$$
\sum_{k=-1}^{1}\left|\hat{\psi}_{2}(\xi+k)\right|^{2}=1, \quad \forall \text { a. e. } \xi \in[-1,1] .
$$

Thus, a classical shearlet $\psi$ is a function which is wavelet-like along one axis and bumplike along another one. Each element $\psi_{a, s, t}$ of classic shearlet has frequency support on a 


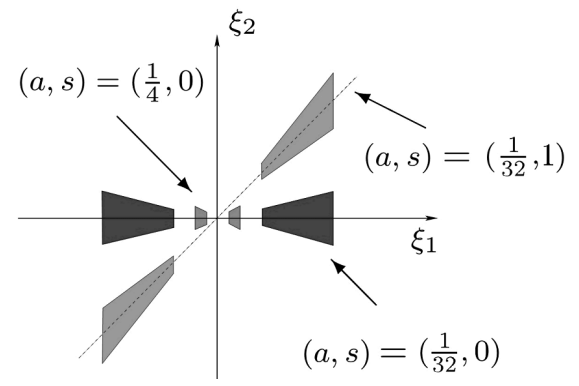

Fig. 2. Support of the classical shearlets $\hat{\psi}_{a, s, t}$ (in the frequency domain) for different values of $a$ and $s$.

pair of trapezoids, at various scales $a$, symmetric with respect to the origin and oriented along a line of slope $s$ (see Fig. 2). Let $\psi \in L^{2}\left(R^{2}\right)$ be an admissible shearlet. Define

$$
C_{\psi}^{+}=\int_{0}^{\infty} \int_{R} \frac{\left|\hat{\psi}\left(\xi_{1}, \xi_{2}\right)\right|^{2}}{\xi_{1}^{2}} d \xi_{2} d \xi_{1}, \quad C_{\psi}^{-}=\int_{-\infty}^{0} \int_{R} \frac{\left|\hat{\psi}\left(\xi_{1}, \xi_{2}\right)\right|^{2}}{\xi_{1}^{2}} d \xi_{2} d \xi_{1}
$$

If $C_{\psi}^{+}=C_{\psi}^{-}=1$, then $\mathcal{S H} \mathcal{H}_{\psi}$ is an isometry.

To obtain the discrete form of the continuous shearlet system and related transform, it is easy to discrete by properly sampling the scale, shear and translation parameters. A (regular) discrete shearlet system, associated with $\psi \in L^{2}\left(R^{2}\right)$ and denoted by $\mathcal{S H}(\psi)$, is defined by

$$
\mathcal{S H}(\psi)=\left\{\psi_{j, k, m}=2^{\frac{3}{4} j} \psi\left(S_{k} A_{2^{j}} \cdot-m\right), j, k \in Z, m \in Z^{2}\right\},
$$

which can be easily obtained by setting $(a, s, t)=\left(2^{-j},-k 2^{-j / 2}, S_{-k 2^{-j / 2}} A_{2^{-j}} m\right)$. Similarly to the continuous case, the discrete shearlet transform of $f \in L^{2}\left(R^{2}\right)$ is defined as the following map

$$
f \rightarrow \mathcal{S H}_{\psi} f(j, k, m)=\left\langle f, \psi_{j, k, m}\right\rangle
$$

where $j, k \in Z, m \in Z^{2}$. The main goal of utilizing shearlet systems is analysis and synthesis of 2-D data, therefore, we need to provide a discrete shearlet system $\mathcal{S H}(\psi)$ which forms a basis or, more generally, a frame. Similar on continuous form, for each $f \in L^{2}\left(R^{2}\right)$, we have the reproducing formula:

$$
f=\sum_{j, k \in Z, m \in Z^{2}}\left\langle f, \psi_{j, k, m}\right\rangle \psi_{j, k, m},
$$

with convergence in the $L^{2}$ sense. Also, in Labate et al. (2005), Guo and Labate (2007) it is illustrated that the classical shearlet is a well-localized function, i.e. it has rapid decay both in the spatial and in frequency domain. The well localization property of classical shearlet implies that discrete shearlet system $\mathcal{S H}(\psi)$ forms a frame. This property is needed 


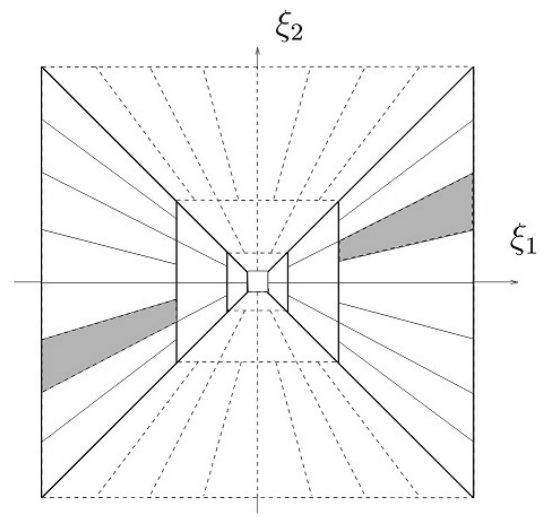

Fig. 3. Tiling of the frequency plane induced by discrete shearlets $\widehat{\psi}_{j, k, m}$. The horizontal region $\mathcal{D}_{h}$ is illustrated in solid line and the vertical region $\mathcal{D}_{v}$ is in dashed line.

for obtaining optimally sparse approximations. Tiling of the frequency plane induced by discrete shearlets $\widehat{\psi}_{j, k, m}$ is shown in Fig. 3 .

Definition 2. The class $\mathcal{E}^{2}\left(R^{2}\right)$ of cartoon-like images is the set of functions $f: R^{2} \rightarrow C$ of the form

$$
f=f_{0}+f_{1} \mathbf{1}_{B},
$$

where $B \subset[0,1]^{2}$ is a set with $\partial B$ being a closed $C^{2}$-curve with bounded curvature, $\mathbf{1}_{B}(x)=\left\{\begin{array}{ll}1, & x \in B \\ 0, & x \notin B\end{array}\right.$ is indicator function and $f_{i} \in C^{2}\left(R^{2}\right)$ are functions with supp $f_{i} \in[0,1]^{2}$ and $\left\|f_{i}\right\|_{C^{2}}=1$ for each $i=0,1$.

The property of optimally sparse approximations of multivariate functions is one of the main motivations to propose the shearlet framework. Before stating the main results, we briefly describe how shearlet expansions are able to achieve optimally sparse approximations. Consider a cartoon-like function $f$ and let $\mathcal{S} \mathcal{H}_{\psi}$ be a discrete shearlet system of (10). For $j \in Z$, the elements of $\mathcal{S} \mathcal{H}_{\psi}$ are approximately inside a box of size $2^{-j / 2} \times 2^{-j}$, it follows that at scale $2^{-j}$ there exists about $O\left(2^{j / 2}\right)$ such waveforms whose support is tangent to the curve of discontinuity. Consequently, for $j$ sufficiently large, each shearlet coefficient $\left\langle f, \psi_{j, k, m}\right\rangle$ can be controlled by Guo and Labate $(2007,2012)$

$$
\left|\left\langle f, \psi_{j, k, m}\right\rangle\right| \leqslant\|f\|_{\infty}\left\|\psi_{j, k, m}\right\|_{L^{1}} \leqslant C 2^{-3 j / 4},
$$

where $C$ is a constant. From inequality (12) and the observation that there exists at most $O\left(2^{j / 2}\right)$ significant coefficients, it can be concluded that the $M$ th largest shearlet coefficient is bounded by $O\left(M^{3 / 2}\right)$. This implies that the following result holds.

Theorem 1. Let $f \in \mathcal{E}^{2}\left(R^{2}\right)$ be a cartoon-like image defined on a bounded domain $\Omega \subset R^{2}$, and let $f_{M}$ be the approximation of $f$ obtained by taking the $M$ largest coefficient 
$\left|\psi_{j, k, m}\right|$ in the shearlets expansion of $f$ given by (11). Then the asymptotic approximation error is given by

$$
\left\|f-f_{M}\right\|^{2} \leqslant C M^{-2}(\log M)^{3}, \quad M \rightarrow \infty .
$$

Proof. To prove see Guo and Labate $(2007,2012)$ and the references therein.

Let $\psi_{j, k, m}$ be a classical shearlet, according to Fig. 2, to attain the reproducing formula (11), it is enough to compute the inner product of $\left\langle f, \psi_{j, k, m}\right\rangle$ in both horizontal region $\mathcal{D}_{h}$ and vertical region $\mathcal{D}_{v}$. In Guo and Labate $(2007,2012)$, for both region index $d=\{h, v\}$, it can be shown that

$$
\left\langle f, \psi_{j, k, m}^{d}\right\rangle=2^{\frac{3 j}{2}} \int_{R^{2}} \hat{f}(\xi) \overline{V\left(2^{-2 j} \xi\right) W_{j, k}^{d}(\xi)} e^{2 \pi i \xi A_{d}^{-j} S_{d}^{-k} m} d \xi
$$

where

$$
\begin{aligned}
& V\left(2^{-2 j} \xi\right)=\hat{\psi}_{1}\left(2^{-2 j} \xi_{1}\right) \chi_{\mathcal{D}_{h}}\left(2^{-2 j} \xi\right)+\hat{\psi}_{1}\left(2^{-2 j} \xi_{2}\right) \chi_{\mathcal{D}_{v}}\left(2^{-2 j} \xi\right), \\
& W_{j, k}^{h}(\xi)= \begin{cases}\hat{\psi}_{2}\left(2^{j} \frac{\xi_{2}}{\xi_{1}}-k\right) \chi_{\mathcal{D}_{h}}(\xi)+\hat{\psi}_{2}\left(2^{j} \frac{\xi_{1}}{\xi_{2}}-k+1\right) \chi_{\mathcal{D}_{v}}(\xi), & \text { if } k=-2^{j} \\
\hat{\psi}_{2}\left(2^{j} \frac{\xi_{2}}{\xi_{1}}-k\right) \chi_{\mathcal{D}_{h}}(\xi)+\hat{\psi}_{2}\left(2^{j} \frac{\xi_{1}}{\xi_{2}}-k-1\right) \chi_{\mathcal{D}_{v}}(\xi), & \text { if } k=2^{j}-1 \\
\hat{\psi}_{2}\left(2^{j} \frac{\xi_{2}}{\xi_{1}}-k\right), & \text { otherwise }\end{cases} \\
& W_{j, k}^{v}(\xi)= \begin{cases}\hat{\psi}_{2}\left(2^{j} \frac{\xi_{2}}{\xi_{1}}-k+1\right) \chi_{\mathcal{D}_{h}}(\xi)+\hat{\psi}_{2}\left(2^{j} \frac{\xi_{1}}{\xi_{2}}-k\right) \chi_{\mathcal{D}_{v}}(\xi), & \text { if } k=-2^{j} \\
\hat{\psi}_{2}\left(2^{j} \frac{\xi_{2}}{\xi_{1}}-k-1\right) \chi_{\mathcal{D}_{h}}(\xi)+\hat{\psi}_{2}\left(2^{j} \frac{\xi_{1}}{\xi_{2}}-k\right) \chi_{\mathcal{D}_{v}}(\xi), & \text { if } k=2^{j}-1 \\
\hat{\psi}_{2}\left(2^{j} \frac{\xi_{1}}{\xi_{2}}-k\right), & \text { otherwise }\end{cases}
\end{aligned}
$$

and $A_{h}=\left(\begin{array}{ll}4 & 0 \\ 0 & 2\end{array}\right), S_{h}=\left(\begin{array}{ll}1 & 1 \\ 0 & 1\end{array}\right)$ and $A_{v}=\left(\begin{array}{ll}2 & 0 \\ 0 & 4\end{array}\right), S_{v}=S_{h}^{T}$. In (13), it is required to compute $\hat{f}(\xi)$ in discrete form, so given an $N \times N$ image $f \in \ell^{2}\left(Z_{N}^{2}\right)$, the 2D discrete Fourier transform (DFT) of $f$ will be:

$$
\hat{f}\left[k_{1}, k_{2}\right]=\frac{1}{N} \sum_{n_{1}, n_{2}=0}^{N-1} f\left[n_{1}, n_{2}\right] e^{-2 \pi i\left(\frac{n_{1}}{N} k_{1}+\frac{n_{2}}{N} k_{2}\right)},
$$

where $-\frac{N}{2} \leqslant k_{1}, k_{2} \leqslant \frac{N}{2}$ and brackets [., .] denote the indices of $\hat{f}$ in Fourier domain. To compute the integrand of (13), in the space domain and at the resolution level $j$, firstly, the Laplacian-pyramid (LP) algorithm (Burt and Adelson, 1983) associated with the pseudopolar Fourier transform (Averbuch et al., 2008) will be utilized. This will achieve the multiscale partition illustrated in Fig. 4, by decomposing $f_{a}^{j-1}\left[n_{1}, n_{2}\right], 0 \leqslant n_{1}, n_{2} \leqslant N_{j-1}$, into a low pass filtered image $f_{a}^{j}\left[n_{1}, n_{2}\right]$, a quarter of the size of $f_{a}^{j-1}\left[n_{1}, n_{2}\right]$, and a high pass filtered image $f_{d}^{j}\left[n_{1}, n_{2}\right]$. Consequently, to compute the shearlet coefficients $\left\langle f, \psi_{j, k, m}^{d}\right\rangle$ given by (13), in the discrete domain, it is enough to compute the inverse 


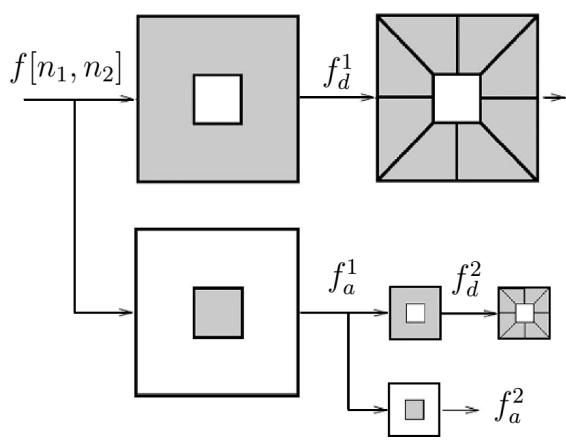

Fig. 4. The figure illustrates the succession of Laplacian-pyramid and directional filtering.

pseudo-polar DFT and apply the inverse two-dimensional fast Fourier transform (FFT) on each decomposition level.

We refer to Labate et al. (2005), Guo and Labate (2007, 2012) for additional information about shearlet and its applications in various sciences and engineering.

\section{Non-Subsampled Shearlet Transform (NSST)}

The main idea of shearlet transform is to filter signals in pseudo-polar grid (Averbuch et al., 2008), and then utilize a bandpass filter in frequency domain directly without sampling operations. Therefore, the directional filtering is kept away from distortion and lead to invariance in shearlet transform. Also, as described in the last section, the discrete form of shearlet transform is achieved by combination of Laplacian-pyramid (Burt and Adelson, 1983) algorithm and directional filter. To improve the computational efficiency and also to reduce the effect of Gibbs phenomena, usually the directional filter is so designed that has a small size support.

The non-subsampled form of Laplacian-pyramid filter (NSLP) is proposed by Cunha et al. (2006). By substituting NSLP for LP and combining it with discrete shearlet transform, non-subsampled shearlet transform (NSST) is designed to improve effectiveness of discrete shearlet transform (Hou et al., 2012). The NSST is known as the shift-invariant version of the shearlet transform. Since the NSST is a fully shift-invariant, multi-scale and multi-directional expansion in comparing to shearlet transform, it can diminish the effect of pseudo-Gibbs phenomena and shearlet-like artifacts in the related processing. The analysis of NSLP can be done as the following iterative processing (Hou et al., 2012):

$$
N S L P_{j+1} f=\left(F h_{j}^{1} \prod_{k=1}^{j-1} F h_{k}^{0}\right) f,
$$

where $f$ is an image, $N S L P_{j+1}$ is the detail coefficients at scale $j+1$, and $F h_{k}^{0}$ and $F h_{j}^{1}$ are low pass and high pass filters of NSLP at scale $j$ and $k$, respectively. Therefore, according to Fig. 4, given an $N \times N$ image $f \in \ell^{2}\left(Z_{N}^{2}\right)$, the procedure of the non-subsampled 


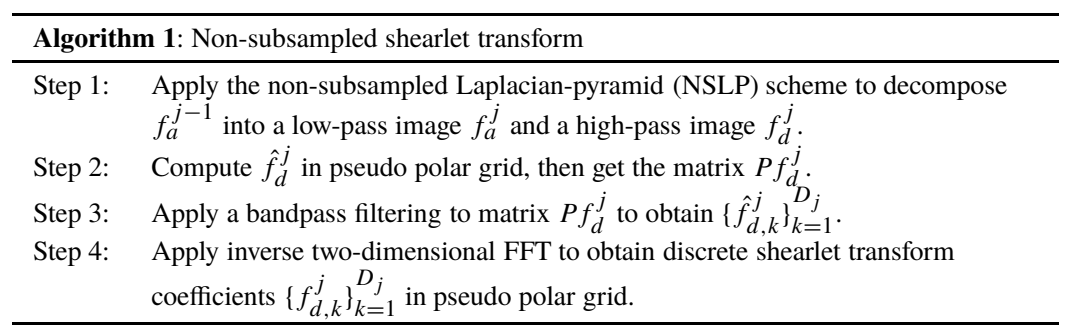

shearlet transform associated with non-subsampled Laplacian-pyramid at a fixed resolution scale $j$ and in the number of direction $D_{j}$ can be summarized in Algorithm 1.

\section{Proposed Algorithm and Experimental Results}

In this section, firstly, we have proposed a denoising algorithm based on non-subsampled shearlet transform associated with log-transform method and secondly evaluated its performance for reducing the speckle noise of the ultrasound images. The structure of present method is similar to those described in Abazari and Lakestani (2018a), Hou et al. (2012). Consider the speckle noisy problem $u=v n_{s}$, where $u$ is observed image, $v$ is noise free image and $n_{s}$ is the multiplicative speckle noise which is independent of noise free image $v$. According to relationships (3)-(6), after applying the log-transform, we have

$$
g=f+\Upsilon,
$$

where $g=\log (u), f=\log (v)$ and $\Upsilon=\log \left(n_{s}\right)$. The additive noise $\Upsilon$ has properties similar to additive Gaussian noise. Our goal is to obtain an estimatation of $f$, namely $\tilde{f}$, from the noisy data $g$ by applying a classical soft thresholding scheme (Labate et al., 2005; Guo and Labate, 2007) on the shearlet coefficients of $g$. The threshold levels are given by $\tau_{j, k}=c_{j} \sigma_{\Upsilon_{j, k}}$, as in Labate et al. (2005), Guo and Labate (2007, 2012, 2013), where $\sigma_{\Upsilon_{j, k}}$ is the standard deviation of noise at scale $j$ and shear directional band $k$ and $c_{j}$ is the scaling parameter. Here the standard deviation $\sigma_{\Upsilon_{j, k}}$ is estimated by using the MATLAB function of std. By using Laplacian-pyramid decomposition, we used five levels of the NSLP decomposition, and we applied a directional decomposition on four of the five scales. According to Fig. 4, we used eight shear filters of size $32 \times 32$ for the first two scales (coarser scales), and sixteen shear filters of size $16 \times 16$ for the third and forth levels (fine scales) and so on. Finally, by using the exp-transformation, the estimated image can be obtained as $\tilde{f}=\exp (\tilde{f})$. Let $f$ be noise free image of size $N \times N$ and $\tilde{f}$ denotes the estimated image, to test our algorithm and to assess its performance, we used two measurements from the following:

- PSNR: as peak signal-to-noise ratio, measured in decibels (dB), defined by

$$
\operatorname{PSNR}(f, \tilde{f})=10 \log _{10} \frac{255 N}{\|f-\tilde{f}\|_{F}},
$$

where $\|\cdot\|_{F}$ is the Frobenius norm. 


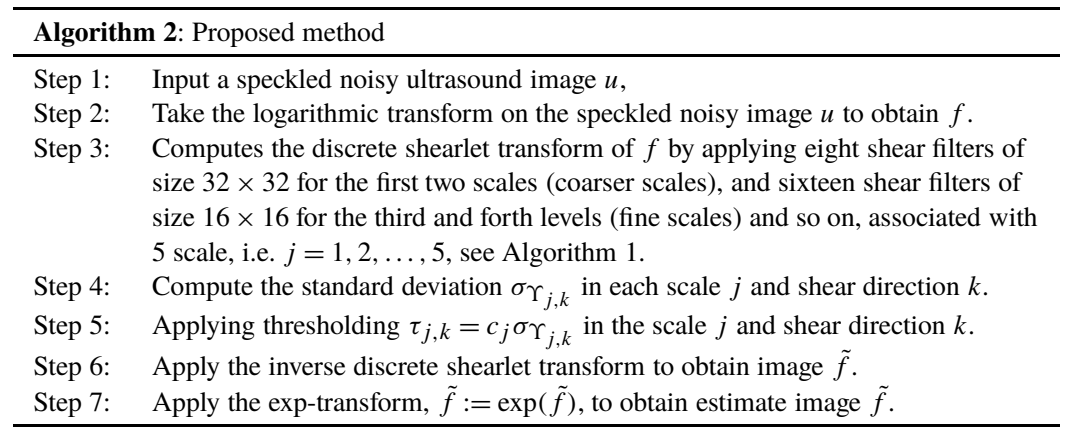

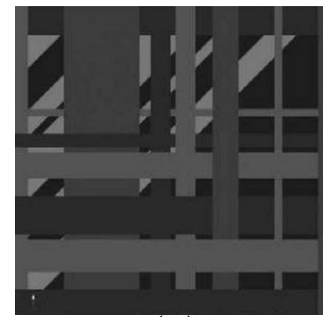

(a)

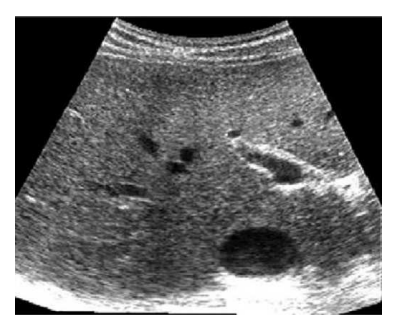

(b)

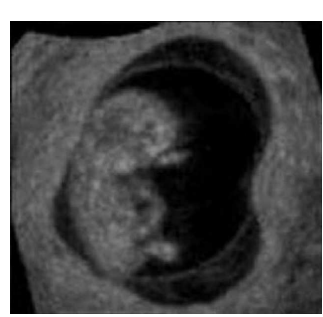

(c)

Fig. 5. Sample test images. (a) Cartoon-like image. (b) Real ultrasound image of a tumour. (c) Real ultrasound image of a fetus.

- SSIM: for calculating the structural similarity (SSIM) index between denoised image $\tilde{f}$ and original image $f$, defined by Wang et al. (2004),

$$
\operatorname{SSIM}(f, \tilde{f})=\frac{\left(2 \mu_{f} \mu_{\tilde{f}}+c_{1}\right)\left(2 \sigma_{f \tilde{f}}+c_{2}\right)}{\left(\mu_{f}^{2}+\mu_{\tilde{f}}^{2}+c_{1}\right)\left(\sigma_{f}^{2}+\sigma_{\tilde{f}}^{2}+c_{2}\right)},
$$

where $\mu_{f}, \mu_{\tilde{f}}$ and $\sigma_{f}^{2}, \sigma_{\tilde{f}}^{2}$ are the average and variance of $f, \tilde{f}$, respectively, $\sigma_{f \tilde{f}}$ is the covariance of $f$ and $\tilde{f}$ and $c_{1}=\left(k_{1} L\right)^{2}, c_{2}=\left(k_{2} L\right)^{2}$ are two variables to stabilize the division with weak denominator. Here, we set $L=255$ as dynamic range of the pixel values and $k_{1}=0.01, k_{2}=0.03$.

The proposed scheme, which is briefly mentioned in Algorithm 2, is implemented using MATLAB 2012. The scours of ShearLab (2008) are used to construct a discrete form of shearlet transform and its non-subsampled form is erected by open source files of NSCT (2008) proposed by Minh. N. Do which initially applied for contourlet transform.

In the first set, we have considered three images listed in Fig. 5 (one sample cartoonlike image and two real ultrasound images same as considered in Yu and Acton (2002), Vese and Osher (2003), Wang et al. (2014), and have compared the results of the proposed method with some related medical ultrasound despeckling techniques from the recent literature such as Yu and Acton (2002), Vese and Osher (2003), Wang et al. (2014). Firstly, 


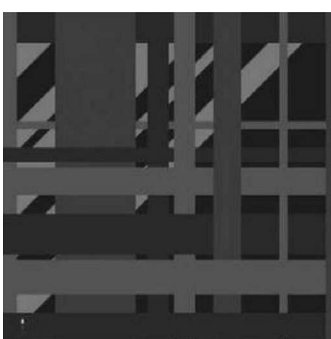

(a)

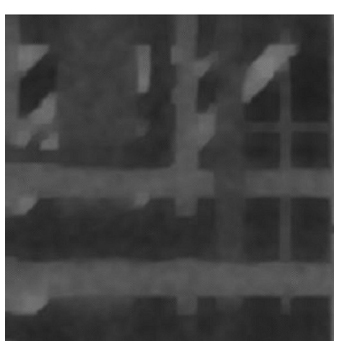

(d)

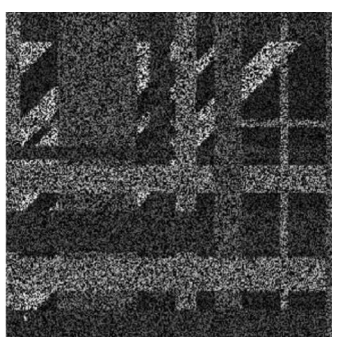

(b)

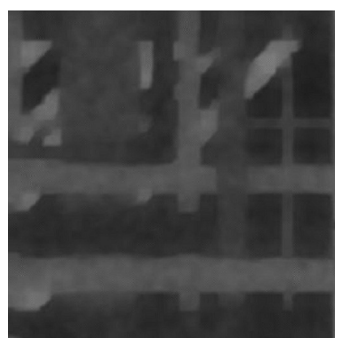

(e)

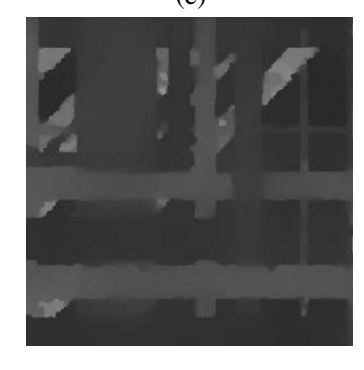

(g)

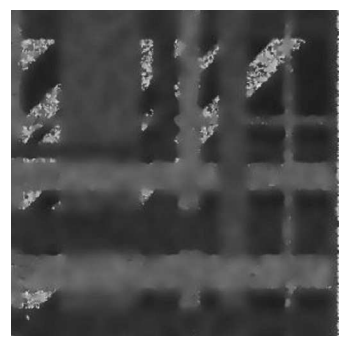

(c)

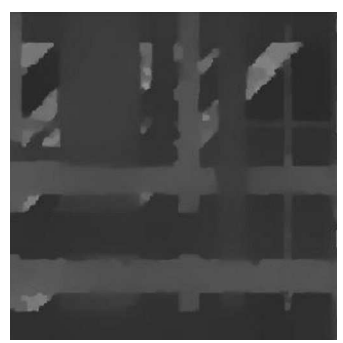

(f)

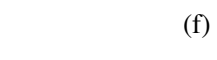




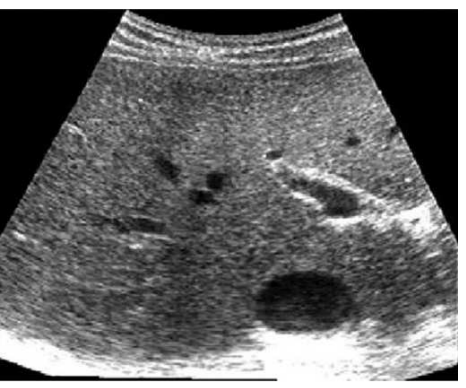

(a)

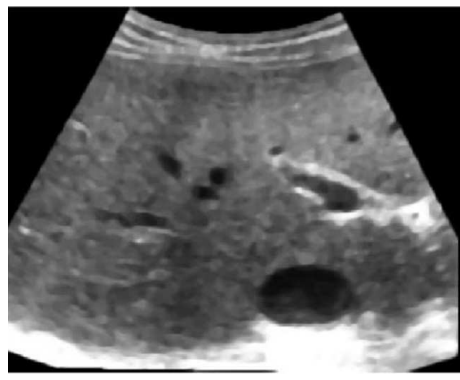

(c)

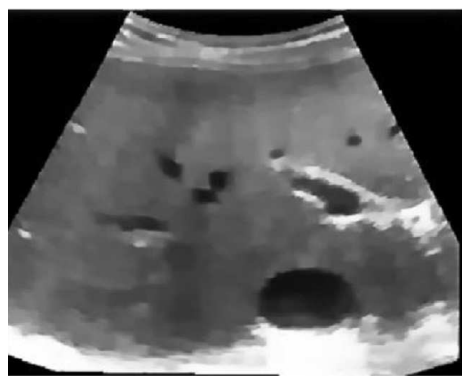

(e)

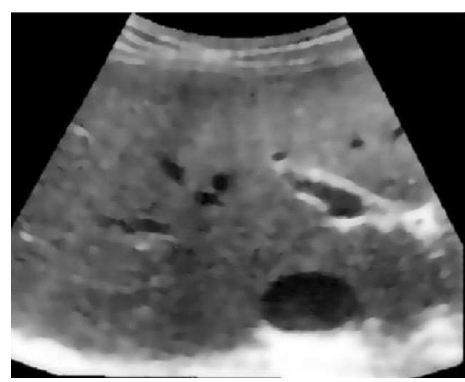

(b)

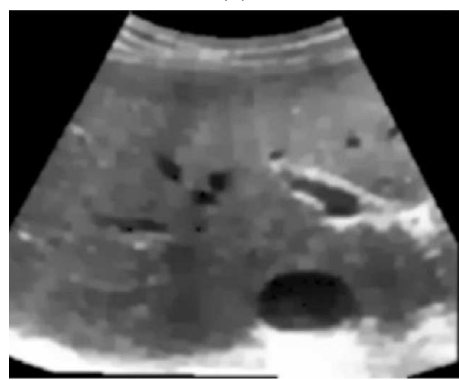

(d)

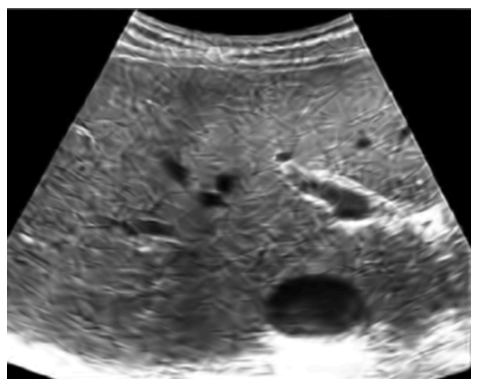

(f)

Fig. 7. Visual comparison of various speckle suppressing methods on a ultrasound image of a tumour. (a) Original image. (b) The denoising result using SRAD (Yu and Acton, 2002). (c) The result using VO method (Vese and Osher, 2003). (d) The denoised result using framelet regularization method without backward diffusion (Wang et al., 2014). (e) The denoised result using framelet regularization method and backward diffusion (Wang et al., 2014). (f) The result of the proposed method.

approach is better than those methods, in addition, the proposed method preserves the texture and edges of images while the methods of Yu and Acton (2002), Vese and Osher (2003), Wang et al. (2014) lead to smooth edges of images. Also, to evaluate the visual quality of the proposed approach, similarly to the methods mentioned in Yu and Acton (2002), Vese and Osher (2003), Wang et al. (2014), we employed the proposed method to two real noisy medical ultrasound images (see Figs. 5(b) and 5(c) same as in Yu and Acton (2002), Vese and Osher (2003), Wang et al. (2014)). The results of our proposed method in comparison with the results of state-of-the-art methods mentioned in $\mathrm{Yu}$ and Acton (2002), Vese and Osher (2003), Wang et al. (2014) are listed in Figs. 7 and 8. 


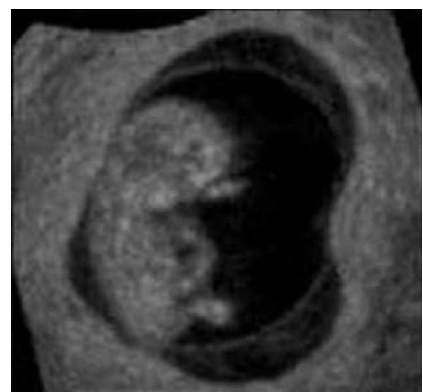

(a)

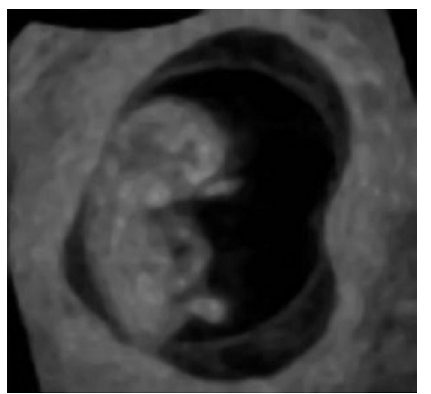

(c)

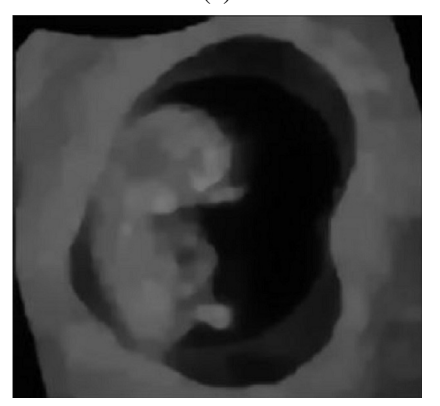

(e)

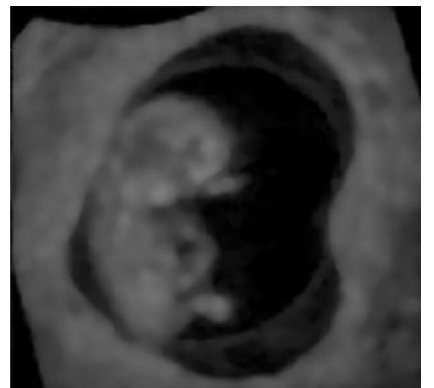

(b)

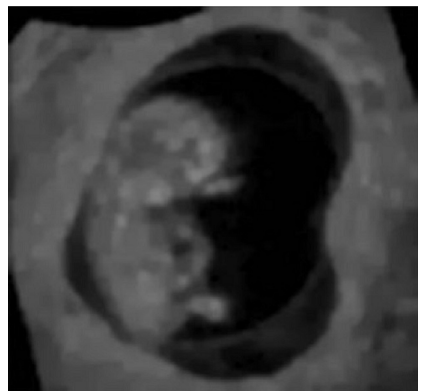

(d)

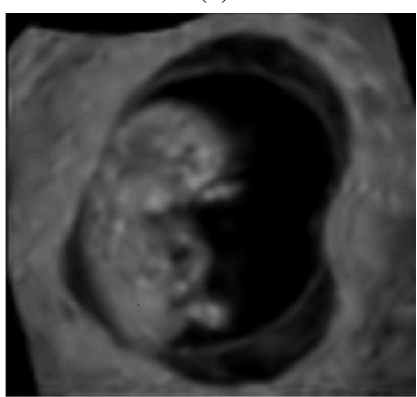

(f)

Fig. 8. Visual comparison of various speckle suppressing methods on a ultrasound image of a fetus. (a) Original image. (b) The denoising result using SRAD (Yu and Acton, 2002). (c) The result using VO method (Vese and Osher, 2003). (d) The denoised result using framelet regularization method without backward diffusion (Wang et al., 2014). (e) The denoised result using framelet regularization method and backward diffusion (Wang et al., 2014). (f) The result of the proposed method.

In the second set, two other real ultrasound images, Fig. 9(a) and 9(b), from Siemens Healthcare (Siemens Healthcare $\mathrm{GmbH}, 2019$ ), are considered and the proposed method is applied to reduce their speckle noise. The despeckled result of these images are shown in Fig. 9(c) and 9(d), respectively, which show that our proposed method can effectively suppress the speckle noises.

Generally, experimental results of the first and the second set of selected images illustrate that the proposed approach can obtain better performance in terms of PSNR and 


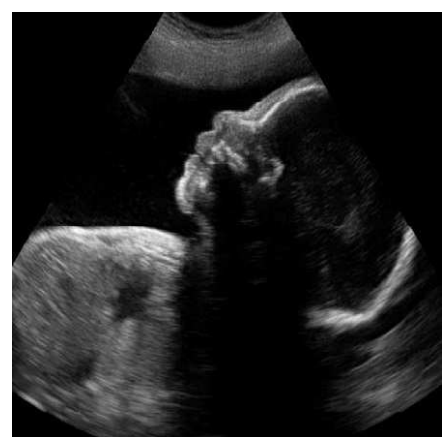

(a)

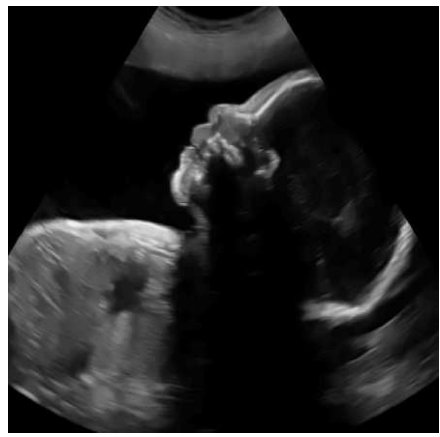

(c)

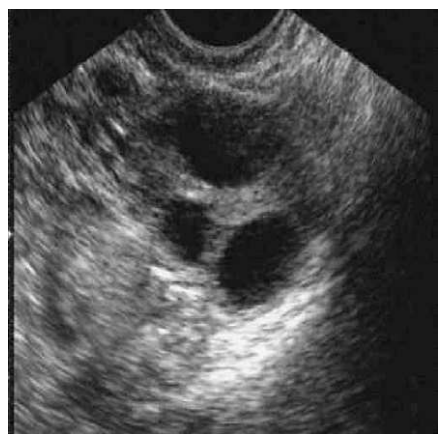

(b)

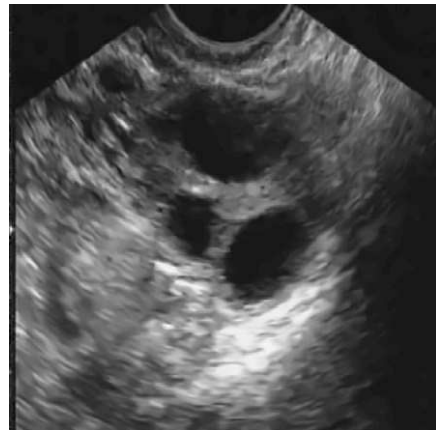

(d)

Fig. 9. Visual comparison of various speckle suppressing methods on two real ultrasound images. (a) and (b) Real ultrasound images. (c) and (d) The result of the proposed method.

SSIM for ultrasound image denoising. From visual comparison, it is easy to see that our proposed method gets smooth effect while preserving the edges of images, which lead to maintain the useful texture information of test images.

\section{Conclusions}

In this paper, we have proposed a denoising method based on discrete shearlet transform and log-transform methods for speckle suppression in ultrasound images. Our numerical tests are done in MATLAB experiments. The experimental results on a sample cartoonlike image and two real ultrasound images polluted by multiplicative speckle noise illustrate the efficiency of the proposed method in terms of qualitative visual evaluation. Experimental results illustrate that the proposed approach can obtain better performance in terms of peak signal to noise ratio (PSNR) and structural similarity index (SSIM) between despeckled images and original images. This approach can be helpful to assist radiologists in their quest and diagnostics. 


\section{References}

Abazari, R., Lakestani, M. (2018a). Fourier based discrete shearlet transform for speckle noise reduction in medical ultrasound images. Current Medical Imaging Reviews, 14(3) 477-483.

Abazari, R., Lakestani, M. (2018b). A hybrid denoising algorithm based on shearlet transform method and Yaroslavsky's filter. Multimedia Tools and Applications, 77(14), 17829-17851.

Abbott, J.G., Thurstone, F.L. (1979). Acoustic speckle: theory and experimental analysis. Ultrason Imaging, 1, 303-324.

Averbuch, A., Coifman, R.R., Donoho, D.L., Israeli, M., Shkolnisky, Y. (2008). A framework for discrete integral transformations I - the pseudo-polar Fourier transform. SIAM Journal on Scientific Computing, 30(2), 764 784 .

Bhuiyan, M.I.H., Ahmad, M.O., Swamy, M.N.S. (2009). Spatially adaptive thresholding in wavelet domain for despeckling of ultrasound images. IET Image Process, 3, 147-162.

Burt, P.J, Adelson, E., (1983). The Laplacian pyramid as a compact image code. IEEE Transactions on Communications, 31(4):532-540.

Candes, E.J. (1998). Ridgelets: Theory and Applications. PhD thesis. Department of Statistics, Stanford University.

Candes, E.J., Donoho, D.L. (2000). Curvelets: a surprisingly effective nonadaptive representation for objects with edges. Tech. report. Department of Statistics, Stanford University.

Colonna, F, Easley, G.R., Guo, K., Labate, D. (2010). Radon transform inversion using the shearlet representation. Applied and Computational Harmonic Analysis, 29(2), 232-250.

Cunha, A.L., Zhou, J., Do, M.N. (2006). The nonsubsampled contourlet transform: theory, design, and applications. IEEE Transactions on Image Processing, 15(10), 3089-3101.

Deka, B., Bora, P.K. (2013). Wavelet-based despeckling of medical ultrasound images. IETE Journal of Research, 59(2), 97-108.

Do, M.N., Vetterli, M. (2005). The contourlet transform: an efficient directional multiresolution image representation. Transactions on Image Processing, 14(12), 2091-2106.

Donoho, D.L., Huo, X. (2001). Beamlets and multiscale image analysis. Tech. report. Stanford University.

Elmoniem, K., Youssef, A., Kadah, A. (2002). Real-time speckle reduction and coherence enhancement in ultrasound imaging via nonlinear anisotropic diffusion. IEEE Transactions on Biomedical Engineering, 49, 997-1014.

Elyasi, I., Pourmina, M.A. (2016). Reduction of speckle noise ultrasound images based on TV regularization and modified bayes shrink techniques. Optik, 127(24), 11732-11744.

Goodman, J.W. (1976). Some fundamental properties of Speckle. Journal of the Optical Society of America, 66 $1145-1150$.

Guo, K., Labate, D. (2007). Optimally sparse multidimensional representation using shearlets. SIAM Journal on Mathematical Analysis, 39, 298-318.

Guo, K., Labate, D. (2012). Optimally sparse representations of 3d data with $c^{2}$ surface singularities using parseval frames of shearlets. SIAM Journal on Mathematical Analysis, 44, 851-886.

Guo, K., Labate, D. (2013). The construction of smooth parseval frames of shearlets. Mathematical Modelling of Natural Phenomena, 8(1), 82-105.

Gupta, S., Chauhan, R.C., Saxena, S.C. (2004). Wavelet-based statistical approach for speckle reduction in medical ultrasound images. Medical \& Biological Engineering \& Computing, 42, 189-192.

Hazarika, D., Nath, V.K., Bhuyan, M. (2015). A lapped transform domain enhanced lee filter with edge detection for speckle noise reduction in sar images. In: IEEE International Conference on Recent Trends in Information Systems (ReTIS), pp. 243-248

Hiremath, P.S., Akkasaligar, P.T., Badiger, S. (2013). Speckle noise reduction in medical ultrasound images, advancements and breakthroughs in ultrasound imaging. G P P Gunarathne, IntechOpen. doi:10.5772/56519.

Hou, B., Zhang, X., Bu, X. (2012). SAR image despeckling based on nonsubsampled shearlet transform. IEEE Journal of Selected Topics in Applied Earth Observations and Remote Sensing, 5(3), 809-823.

Huang, Y., Hu, B., Zhu, J. (2016). Study on the use of quantitative ultrasound evaluation of diabetic neuropathy in the rat sciatic nerve. Australasian Physical \& Engineering Sciences in Medicine, 39(4), 997-1005.

Kabir, S.M., Bhuiyan, M.I.H. (2015). Modeling of log-transformed speckle noise in the contourlet transform domain for medical ultrasound images. Journal of Circuits, Systems, and Computers, 24(2), 1540004 (35 pp.).

Khare, A., Khare, M., Jeong, Y., Kim, H., Jeon, M. (2010). Despeckling of medical ultrasound images using Daubechies complex wavelet transform. Signal Processing, 90(2), 428-439. 
Krissian, K., Westin, C., Kikinis, R., Vosburgh, K. (2007). Oriented speckle reducing anisotropic diffusion. IEEE Transactions on Image Processing, 16, 1412-1424.

Kutyniok, G., Lim, W. (2011). Image separation using wavelets and shearlets. In: Curves and surfaces, Avignon, France, 2010, Lecture Notes in Computer Science, Vol. 6920.

Labate, D., Lim, W.-Q., Kutyniok, G., Weiss, G. (2005). Sparse multidimensional representation using shearlets. In: Wavelets XI, San Diego, CA, 2005. SPIE Proc., Vol. 5914. SPIE, Bellingham, WA, pp. 254-262.

Lakestani, M., Razzaghi, M., Mousavi, Z. (2016). Combined shearlet shrinkage and total variation minimization for image denoising. Iranian Journal of Science and Technology, Transactions A: Science. doi:10.22099/IJSTS.2016.3677.

Loupas, T., Mcdicken, W., Allan, P.L. (1989). An adaptive weighted median filter for speckle suppression in medical ultrasonic images. IEEE Transactions on Circuits and Systems, 36(1), 129-135.

Meyer, F.G., Ronald, R.C. (1997). Brushlets: a tool for directional image analysis and image compression. Applied and Computational Harmonic Analysis, 4(2), 147-187.

Non-subsampled contourlet transform (2008). MATLAB source, Retrieved from http://http://minhdo.ece.illinois.edu/software/.

Patel, V.M., Easley, G., Healy, D.M. (2009). Shearlet-based deconvolution. IEEE Transactions on Image Processing, 18, 2673-2685.

Ragesh, N.K., Anil, A.R., Rajesh, R. (2011). Digital image denoising in medical ultrasound images: a survey. In: ICGST AIML-11 Conference, Dubai, UAE, pp. 12-14.

Rajeshwar, D. (2018). Speckle noise reduction of ultrasound images using BFO cascaded with wiener filter and discrete wavelet transform in homomorphic region. Procedia Computer Science, 132, 1543-1551.

Ritenour, E.R., Nelson, T.R., Raff, U. (1984). Application of median filter to digital readiographic images. Proc. 7th International Conference on Acoustics, Speech and Signal Processing, 23, 1-4.

ShearLab (2008). Computer software, MATLAB source. Retrieved from http://www.shearlet.org.

Siemens Healthcare GmbH (2019). Available: http://www.healthcare.siemens.com/ultrasound.

Vard, A., Jamshidi, K., Movahhedinia, N. (2012). An automated approach for segmentation of intravascular ultrasound images based on parametric active contour models. Australasian Physical \& Engineering Sciences in Medicine, 35(2), 135-150.

Vese, L., Osher, S. (2003). Modeling textures with total variation minimization and oscillating patterns in image processing. Journal of Scientific Computing, 19(11), 553-572.

Vishwakarma, A., Bhuyan, M.K., Iwahori, Y. (2018) Non-subsampled shearlet transform-based image fusion using modified weighted saliency and local difference. Multimedia Tools and Applications, 77(24), 3201332040 .

Wang, G., Xu, J., Pan, Z., Diao, Z. (2014). Ultrasound image denoising using backward diffusion and framelet regularization. Biomedical Signal Processing and Control, 13, 212-217.

Wang, Z., Simoncelli, E.P., Bovik, A.C. (2004). Multiscale structural similarity for image quality assessment. Conference Record of the Thirty-Seventh Asilomar Conference on Signals, Systems and Computers, 2(2), 1398-1402.

Yu, Y., Acton, S. (2002). Speckle reducing anisotropic diffusion. IEEE Transactions on Image Processing, 11 (2002) $1260-1270$.

Zong, X., Laine A.F., Geiser, E.A. (1998). Speckle reduction and contrast enhancement of echocardiograms via multiscale nonlinear processing. IEEE Transactions on Medical Imaging, 17, 532-540.

Zhang, Y., Wang, Y., Wang, W., Liu, B. (2001). Doppler ultrasound signal denoising based on wavelet frames. IEEE Transactions on Ultrasonics, Ferroelectrics, and Frequency Control, 48, 709-716. 
R. Abazari received the MS degree in applied mathematics from University of Mohaghegh Ardabili, Ardabil, Iran, in 2008 and PhD degree in applied mathematics in 2017 from University of Tabriz, Tabriz, Iran. He is now an adjunct lecturer in applied mathematics at University of Mohaghegh Ardabili since September 2009. He is also an research associate at the Young Researchers and Elite Club, Ardabil, Iran. His research interests include mathematical method in physics, medical sciences and image analysis.

M. Lakestani received the BS degree in applied mathematics from University of Tabriz, Iran, in 1998 and the MSc and PhD degree both in applied mathematics from Amirkabir University of Technology - Tehran Polytechnic, Tehran, Iran, in 2000 and 2005, respectively. Since 2005, he is a professor at University of Tabriz. His research interests include numerical analysis, with special emphasis on wavelet theory, time-frequency analysis and image processing. 